\title{
Pilot study of Ixodes ricinus ticks preference for human ABO blood groups using a simple in vitro method
}

\author{
Alena Žákovská',2,A-F, Jan Janeček ${ }^{1, C-F}$, Helena Nejezchlebová',A,C,E , Hana Lya Kučerová1,B-C \\ ${ }^{1}$ Department of Animal Physiology and Immunology, Faculty of Science, Masaryk University, Brno, Czech Republic \\ 2 Department of Biology, Faculty of Education, Masaryk University, Brno, Czech Republic \\ A - Research concept and design, B - Collection and/or assembly of data, C - Data analysis and interpretation, \\ $D$ - Writing the article, $E$ - Critical revision of the article, $F$ - Final approval of article
}

Žákovská A, Janeček J, Nejezchlebová H, Kučerová HL. Pilot study of Ixodes ricinus ticks preference for human ABO blood groups using a simple in vitro method. Ann Agric Environ Med. 2018; 25(2): 326-328. doi: 10.26444/aaem/85167

\begin{abstract}
Introduction and objectives. The existence of a blood group preference for ticks is a problem widely discussed among the lay public but often neglected by the scientific community. The Ixodes ricinus tick transmits serious zoonotic diseases such as Lyme borreliosis, tick encephalitis, or anaplasmosis. The preventive strategies include vaccination (if available) and individual measures including the use of repellents and avoidance of risk areas.

Materials and method. Since this topic is relatively neglected in the scientific literature, a simple in vitro method was used. Ticks used in this study were collected in the suburban region of Ruda in Brno, Czech Republic. One hundred active nymphs of the collected ticks were tested for preferences for blood groups, using Petri dishes and blood samples from volunteers. To demonstrate the threat of ticks and the diseases they transmit, the positivity of one of the most abundant zoonosis, Lyme borreliosis, was tested using dark-field microscopy.

Results. The results obtained showed that the examined ticks were attracted most by blood group $A$, whereas the least preferred was group $B$, which was proved statistically $(p<0.05)$. The mean positivity of collected ticks for the presence of spirochaetes was $9.35 \%$.

Conclusions. The results indicate that the host selection of ticks may be influenced by the physiological or biochemical profile of an individual, such as their blood group. This means that a blood group of an individual can be one of the factors that increase the risk of tick bite and the transmission of dangerous diseases and thus must not be underestimated.
\end{abstract}

\section{Key words}

in vitro, Lyme borreliosis, spirochaetes, vector

\section{INTRODUCTION}

Ticks are one of the most important vectors of pathogens that cause diseases in both humans and animals. They are considered to be the second most important vector of human diseases after mosquitoes [1]. They transmit various severe diseases, e.g. Lyme borreliosis (LB), tick-borne encephalitis, anaplasmosis, babesiosis, etc. [2, 3]. These tick-transmitted diseases, especially LB, represent a growing health problem in the Czech Republic and other countries in Europe. This study focuses on the most common species of ticks in Europe as well as in the Czech Republic, Ixodes ricinus, which is also the primary vector of Borrelia burgdorferi sensu lato, the etiologic agent of LB [4].

The ticks preference for certain blood groups is a question which is still not scientifically answered but abundantly discussed among the general public on the Internet or in everyday conversation. The influence of blood groups on certain diseases such as malaria or some cancers has been already discussed and proved [5]. The best described is the link between the milder progress of malaria transmitted by mosquitoes and blood type O. This may suggest that there

Address for correspondence: Jan Janeček, Department of Animal Physiology and Immunology, Faculty of Science, Masaryk University Brno, Kamenice 5, BrnoBohunice, 62500 Brno, Czech Republic

e-mail: 424154@mail.muni.cz

Received: 23.07.2017; accepted: 08.02.2018; first published: 23.03.2018 could be a similar relationship between tick-borne diseases and some blood group(s), or the evolutionary preferences between the arthropod vector and hosts with different blood groups.

\section{OBJECTIVE}

The aim of this pilot study was to determine the ticks preference for blood groups using a simple in vitro method that eliminates the need for animal models and possible ethical problems. The measured results were statistically evaluated.

\section{MATERIALS AND METHOD}

Ixodes ricinus ticks were collected in the locality of Ruda near Brno Reservoir, a frequently visited place, by flagging for an hour at the same time once every two weeks. Collection was by dragging a white flannel flag over the low vegetation.

Only nymphs were used due to their higher numbers and better activity. Before the examination, all nymphs were tested for their activity - each tick was placed individually on a heated vertical plastic cylinder covered with filter paper, simulating the mammalian environment. Only ticks which showed negative geotaxis (i.e. they moved upwards) 
were considered to be attracted by the host and used for the experiment. In this way, the total number of one hundred nymphs was selected.

The preferences of ticks for human blood groups were examined with a simple method using sterile Petri dishes. Samples of blood were obtained from healthy volunteers whose blood type had been previously determined. Blood sample collection was performed by a finger prick. A drop of blood of every blood type (A, B, AB or O) was applied to a sterile layer of filter paper on a Petri dish. The distances between individual drops were the same. The tick was placed in the middle of a dish. One hundred nymphs were used, as described above. Which blood drop the tick chooses at the beginning of a test (at the time 0 ) was determined, and then where the tick located after one minute and after two minutes. The simplicity of this in vitro method partly eliminates the influence of factors other than the blood itself.

After the experiment, all ticks were also examined for the presence of spirochaetes using dark-field microscopy to show the potential risk of transmission of zoonotic pathogens.

Statistical analysis. Analysis of statistical data was performed by means of software STATISTICA 10 (StatSoft, 2011). A chisquared test was used to evaluate the potential influence of blood groups on the behaviour of ticks. These numbers were compared with each other using homogenous groups (Fisher's LSD test). Data with p-value less than 0.05 (i.e. 5\%) has been considered as statistically significant.

\section{RESULTS}

The first blood group which a tick chose (at the time $\mathrm{t}=0$ ), was most often group A (36\%), followed type O (32\%), AB (17\%), then group B (15\%). The preference for blood groups A and $B$ decreased with time $(\mathrm{p}=0.0128$ and 0.0004 , respectively), while the preferences for groups $\mathrm{AB}$ and $\mathrm{O}$ did not vary significantly with time ( $\mathrm{p}=0.2670$ and 0.3396 , respectively). It can be stated that the most statistically preferred was blood group A $(\mathrm{p}<0.0001)$, followed by the second groups $-\mathrm{O}$ $(\mathrm{p}<0.0001)$ and $\mathrm{AB}(\mathrm{p}<0.0001)$, and the least preferred was blood group B $(\mathrm{p}<0.0001)$ (Fig. 1).

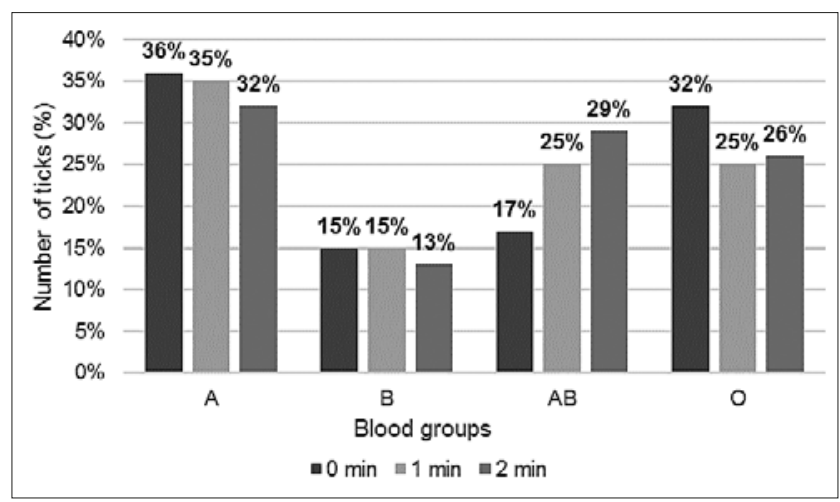

Figure 1. Percentage of ticks attracted by a blood drop of a particular blood group and its changes in time

From the total of 321 ticks (64 larvae, 236 nymphs, 14 males and 7 females) collected every second week, 42 samples were positive for the presence of spirochaetes, which means $9.35 \%$ positivity. The nymphs show an infectivity value of $11.02 \%$.

\section{DISCUSSION}

From the information obtained from the results, it can be noted that all collected ticks were also tested for the presence of pathogenic spirochaetes, within the framework of the regular monitoring prevalence of Borrelia burgdorferi sensu lato at the selected location. The infectivity shows a value of $9.35 \%$. In comparison to other studies undertaken in the Czech Republic and Slovakia, the positivity from Ruda is lower. These studies reveal an average infectivity of $14.1 \%$ [6] and 20.4\% [7], whereas a study by Venclíková et al. [8] revealed the minimal positivity rate to be between $13.8 \%$ - $15 \%$ in suburban and natural areas of Ostrava, the third largest city in the Czech Republic.

No relevant information about preferences of ticks for blood groups exists in the scientific literature. However, the preferences to blood groups have been previously tested in mosquitoes. Shirai et al. demonstrated that humans with blood group $\mathrm{O}$ attracted more Aedes albopictus mosquito than other groups [9]. They also found that $\mathrm{H}$ antigen attracted mosquitoes more than A and B antigens. Anjomruz et al. demonstrated a significantly higher blood preference of Anopheles stephensi for $\mathrm{AB}$ group than to the other groups [10]. Both research teams used human volunteers to study the preferences. In one case, they allowed the mosquitos to feed on the exposed hands of volunteers. In the second study, the experimenters were studying only 'landing'preferences of mosquitos with amputated proboscis. These approaches are ethically and otherwise problematic; therefore, for the current study it was decided to use only blood samples.

The results obtained revealed that the statistically significant preferred blood group was group A, while the least preferred was group B, the preference for which decreased with time. The reason for other studies showing different preferred groups is multifactorial. Some mosquito species are the vector of malaria; it is known that blood group $\mathrm{O}$ is protective against severe malaria, while others are not. This could be the reason why some species of mosquitoes in affected areas prefer other blood groups than type O. On the other hand, blood type $\mathrm{O}$ is highly prevalent in Africa, which could cause the host preferences of some species of mosquitos for this blood group in humans [10].

The advantages of the in vitro approach used in the current research, namely, its simplicity and the suppression of unwanted factors, is also its weakness. The living organism is a complex system of numerous elements which interact with each other, therefore, the effect of the blood group on ticks feeding preferences in the the living organism could be influenced by other factors.

Hence, a future study using in vivo approach will be required. The authors cannot suggest using model animals due to the fact that different animal species have different systems of blood groups. One possibility to overcome this problem could be to use human volunteers. However, this approach involves increased risk of contracting a tick-borne infection. As mentioned above, one possibility is to amputate the proboscis of the vector and/or use ticks which have been both bred and kept from eggs in sterile conditions to avoid the risk of carrying infectious agents. 


\section{CONCLUSION}

The presented study demonstrated that blood group might be one of the factors determining the feeding preferences of Ixodes ricinus ticks. The information obtained about the potential preference of ticks for specific blood groups can be used to reduce the risk of tick bite: people with the risk blood type A should take appropriate measures to protect themselves more effectively, and decrease the risk of contracting dangerous zoonotic diseases transmitted by ticks.

\section{Acknowledgments}

This study was supported by the Specific Research Programme at Masaryk University in Brno, Czech Republic. Authors declare they have no conflict of interest regarding this article.

\section{REFERENCES}

1. Beugnet F, Marié JL. Emerging arthropod-borne diseases of companion animals in Europe. Vet Parasitol. 2009; 163(4): 298-305.
2. Service MW. The Encyclopedia of Arthropod-transmitted Infections. CABI, Wallingford, 2001.

3. Hubálek Z, Rudolf I. Microbial Zoonoses and Sapronoses. Springer Netherlands, 2011.

4. Vassallo M, Pérez-Eid C. Comparative behavior of different life-cycle stages of Ixodes ricinus (Acari: Ixodidae) to human-produced stimuli. J Med Entomol. 2002; 39(1): 234-236.

5. Franchini M, Bonfanti C. Evolutionary aspects of ABO blood group in humans. Clin Chim Acta. 2015; 444: 66-71.

6. Pet'ko B, Štefančíková A, Tresová G, Peterková J, Škardová I, Prokopčáková H, Čisláková L. Castor bean tick (Ixodes ricinus) as a source of infection of humans and dogs, and a vector of Lyme disease. Slovenský veterinársky časopis. 1996; 21: 313-319.

7. Hubálek Z, Halouzka J, Juřicová Z. Investigation of haematophagous arthropods for Borreliae summarized data, 1988-1996. Folia Parasitol. 1998; 45(1): 67-72.

8. Venclíková K, Betášová L, Sikutová S, Jedličková P, Hubálek Z, Rudolf I. Human pathogenic borreliae in Ixodes ricinus ticks in natural and urban ecosystem (Czech Republic). Acta Parasitol. 2014; 59(4): 717-720.

9. Shirai Y, Funada H, Takizawa H, Seki T, Morohashi M, Kamimura K. Landing preference of Aedes albopictus (Diptera: Culicidae) on human skin among $\mathrm{ABO}$ blood groups, secretors or nonsecretors, and $\mathrm{ABH}$ antigens. J Med Entomol. 2004; 41(4): 796-799.

10. Anjomruz M, Oshaghia MA, Pourfatollah AA, Sedaghat MM, Raeisi A, Vatandoost H, et al. Preferential feeding success of laboratory reared Anopheles stephensi mosquitoes according to ABO blood group status. Acta Trop. 2014; 140: 118-123. 